\title{
New approach to large-scale nuclear structure calculations
}

\author{
J. Dukelsky ${ }^{1}$ and S. Pittel ${ }^{2}$ \\ ${ }^{1}$ Instituto de Estructura de la Materia, Consejo Superior de Investigaciones Cientificas, Serrano 123, E-28006 Madrid, Spain \\ ${ }^{2}$ Bartol Research Institute, University of Delaware, Newark, Delaware 19716
}

(Received 22 January 2001; published 2 May 2001)

\begin{abstract}
A new approach to large-scale nuclear structure calculations, based on the density matrix renormalization group (DMRG), is described. The method is tested in the context of a problem involving many identical nucleons constrained to move in a single large- $j$ shell and interacting via a pairing plus quadrupole interaction. In cases in which exact diagonalization of the Hamiltonian is possible, the method is able to reproduce the exact results for the ground-state energy and the energies of low-lying excited states with extreme precision. Results are also presented for a model problem in which exact solution is not feasible.
\end{abstract}

DOI: 10.1103/PhysRevC.63.061303

PACS number(s): 21.60.Cs, 05.10.Cc

The nuclear shell model [1] is arguably the most powerful approach for a microscopic description of nuclear properties. In this approach, the low-energy structure of a given nucleus is described by assuming an inert doubly-magic core and then seeing how the effective interaction scatters the remaining nucleons over the valence orbits of the next major shell(s). Despite the enormous simplification provided by this shell-model approach, it is still only possible to describe nuclei in this way within limited regions of the periodic table, namely where the number of active nucleons or the degeneracy of the valence shells is sufficiently small. The most ambitious implementation of this method to date has been in a treatment of the binding energies of nuclei in the $f p$ shell through ${ }^{64} \mathrm{Zn}[2]$.

If we wish to use the shell-model approach in the description of heavier nuclei or nuclei further from closed shells, we must come up with a reliable truncation procedure, one that can reduce the number of shell-model configurations while maintaining the key dynamics of the interacting nucleons. Historically, many approaches have been used. Some truncate on the basis of weak-coupling considerations [3], others on the basis of symmetry considerations [4], and others on the basis of Monte Carlo sampling [5]. Within the latter approach, it has recently proven possible to go beyond the $f p$ shell to describe the transition from spherical to deformed nuclei in the barium isotopes [6]. In this work, we describe an alternative approach to large-scale nuclear structure calculations, called the density matrix renormalization group (DMRG). This method, developed originally in the framework of low-dimensional quantum lattice systems [7], was recently extended to finite Fermi systems [8]. The new methodology was first used in the treatment of a pairing problem [8] of relevance to the physics of ultrasmall superconducting grains. Here we begin to explore its potential for use in nuclear structure.

The basic idea of the DMRG method, as appropriate to finite Fermi systems, is to systematically take into account the physics of all single-particle levels. This is done by first taking into account the most important levels, namely those that are nearest to the Fermi surface, and then gradually including the others in subsequent iterations. At each step of the procedure, truncation is implemented so as to optimally take into account the effects of the levels that are added while keeping the problem tractable.

We will assume from the outset that each single-particle level in the problem admits the same number of possible states. This is the case, for example, when working in a single-particle basis of axially-symmetric Nilsson-like levels, or if we consider pairs of time reversal states in a spherical basis. Each such level can accommodate four possible states, one with no particles, two with one particle, and one with two particles. We denote the number of states that a given single-particle level admits as $s$; in this case, $s=4$. We will explain later the rationale for this simplifying assumption, after we have described the procedure more fully.

Next, we assume that we have already treated some of the levels for particles and the same number for holes, namely those closest to the Fermi energy, and that the number of states in the two spaces is the same. We call that common number of states $m$. When we add the next particle level and the next hole level, the number of particle states increases from $m$ to $s \times m$ and the number of hole states likewise increases from $m$ to $s \times m$. The DMRG method truncates from the $s \times m$ states to the optimum $m$ states, both for particles and for holes.

Following this optimal truncation, we then add the next levels for particles and holes and truncate again to the optimum $m$ states for each. This procedure is continued until all particle and hole levels have been sampled. (Note: If one type of level is exhausted before the other, we subsequently add those levels that remain one at a time.)

Finally, we carry out the calculation as a function of $m$, the number of particle and hole states that are kept. All previous applications of the DMRG method [9] have exhibited exponential convergence of the results (e.g., for the groundstate energy) as a function of $m$, suggesting that this should likewise be the case in applications to nuclear structure. If so, we stop the procedure once the changes that arise with increasing $m$ are acceptably small.

The key question not yet addressed is "What do we mean by optimum and how do we implement a truncation to those optimum states?"

To answer these questions, we now consider the ground state of the full system, expressed as a sum of terms involving states in the particle space, $|i\rangle_{P}$, coupled to states in the hole space, $|j\rangle_{H}$, 


$$
|\Psi\rangle=\sum_{i=1, N_{P}} \sum_{j=1, N_{H}} \Psi_{i j}|i\rangle_{P}|j\rangle_{H}
$$

where $N_{P}$ is the dimension of the particle space and $N_{H}$ is the corresponding dimension of the hole space. (In the earlier discussion, $N_{P}=N_{H}=s \times m$.) Note that we only need consider states for which the total angular momentum projection is 0 and for which the total number of particles and holes is the same.

What we would like to do is to construct the optimal approximation to the ground-state wave function $|\Psi\rangle$ that is achieved when we only retain $m$ states in the particle space and $m$ states in the hole space. By optimal, we will mean that the projected wave function, the one that arises following the truncation, has the largest possible overlap with the exact ground-state wave function $|\Psi\rangle$.

We will implement the truncation in two steps, first asking what is the best approximation when we truncate the particle states and then what is the best approximation when we truncate the hole space.

To arrive at the optimum truncation for particles, we first introduce the corresponding ground-state reduced density matrix,

$$
\rho_{i i^{\prime}}^{P}=\sum_{j=1, N_{H}} \Psi_{i j} \Psi_{i^{\prime} j}^{*}
$$

obtained by contracting over all the states of the hole space. We then diagonalize this $N_{P} \times N_{P}$ matrix,

$$
\rho^{P}\left|u^{\alpha}\right\rangle_{P}=\omega_{\alpha}^{P}\left|u^{\alpha}\right\rangle_{P}
$$

A given eigenvalue $\omega_{\alpha}^{P}$ represents the probability of finding the particle state $\left|u^{\alpha}\right\rangle_{P}$ in the full ground-state wave function of the system. The optimal truncation corresponds to retaining the $m$ eigenvectors that have the largest probability of being present in the ground state wave function, or equivalently those that correspond to the largest eigenvalues $\omega_{\alpha}^{P}$ [9].

Analogously, we construct the ground-state reduced density matrix for holes,

$$
\rho_{j j^{\prime}}^{H}=\sum_{i=1, N_{P}} \Psi_{i j} \Psi_{i j^{\prime}}^{*}
$$

by contracting over particle states. If we diagonalize the $N_{H} \times N_{H}$ density matrix for holes and retain only the $m$ states with the largest eigenvalues, we are guaranteed to be choosing the best hole truncation in the sense of maximal overlap with the exact ground state.

Summarizing, in each DMRG iteration we add to the system a new particle level and a new hole level. We then construct the Hamiltonian matrix for the enlarged system and diagonalize it for the ground state and some low-lying excited states. From the ground-state wave function, we calculate the reduced density matrices for particles (2) and holes (4) and diagonalize them. For each, we retain the $m$ eigenvectors corresponding to the $m$ largest eigenvalues.
The final step in a given iteration involves transforming all operator matrices from the enlarged particle and hole spaces to the smaller $(m \times m)$ spaces. More specifically, we transform the matrices of $a^{\dagger}, a^{\dagger} a, a^{\dagger} a^{\dagger}, a^{\dagger} a^{\dagger} a$, and $a^{\dagger} a^{\dagger} a a$. When we proceed to the next iteration, in which extra levels are added, the resulting Hamiltonian matrices can be readily obtained since we have available the relevant operator matrices in the smaller spaces while those of the conjugate operators (e.g., $a$ is conjugate to $a^{\dagger} a^{\dagger} a$ ) in the space of the extra orbits are very easy to evaluate.

As noted earlier, the first application of this methodology was reported in Ref. [8] in the context of a pairing Hamiltonian acting over a very large number of equally separated doubly-degenerate single-particle levels. The Hamiltonian for this so-called picket fence model is

$$
H=\sum_{j, \sigma=+,-} \epsilon_{j \sigma} c_{j \sigma}^{\dagger} c_{j \sigma}-\lambda d \sum_{j, j^{\prime}} c_{j+}^{\dagger} c_{j-}^{\dagger} c_{j^{\prime}-} c_{j^{\prime}+},
$$

with $\epsilon_{j \sigma}=j d$ and $j=1, \ldots, \Omega$. Here, $\Omega$ is the total number of levels and $d$ is the spacing between adjacent levels The calculations of Ref. [8] assumed half filling, so that the total number of particles distributed over the $\Omega$ levels is also $\Omega$.

As an example of the quality of the results that can be obtained with this method, consider the case of $\Omega=400$. For this value of $\Omega$, the dimension of the full Hamiltonian matrix that would have to be diagonalized is $10^{119}$. While this problem is obviously much too large to be solved by standard diagonalization techniques, it can be solved to arbitrary accuracy using a method developed by Richardson in the 1960s [10]. For a problem in which $\lambda=0.224$, the Richardson solution for the ground state has a correlation energy of -22.5183141 in units of $d$. When the same problem is solved using the DMRG method with $m=60$, a ground state correlation energy of -22.5168 in the same units is achieved. The agreement is to better than 1 part in $10^{4}$, despite the fact that the maximum dimension Hamiltonian matrix that had to be treated for this value of $m$ was only 3066 .

Clearly, for a pairing Hamiltonian acting in a uniform doubly-degenerate single-particle space, the density matrix renormalization group method works remarkably well. On the other hand, the Hamiltonian (5) of this problem is extremely simple, being equivalent to a one-body Hamiltonian for a hard-core boson. In that respect, even though the previous results are suggestive that the method might also work well for problems in nuclear structure physics, we still need to demonstrate this more convincingly by applying it to a fermion problem with a true two-body interaction.

With that in mind, we have now completed the first test of the new DMRG methodology in nuclear structure. We considered a schematic model in which a large number of identical particles are restricted to a single large- $j$ shell and interact via a sum of a pairing plus quadrupole force. Unfortunately, for a single- $j$ shell, the $0^{+}$ground state has equal population of all $m$ substrates and thus such a model does not accommodate a Fermi surface. Since this is not the case for realistic problems, in which there is invariably a Fermi surface, we force one on the current problem by add- 
TABLE I. Ground-state energy for ten particles in a $j=25 / 2$ level. The Hamiltonian parameters are $x=1, g=0$, and $\epsilon=0.1$.

\begin{tabular}{ccc}
\hline \hline$m$ & $\begin{array}{c}E_{\text {g.s. }} \\
\text { Exact }\end{array}$ & $\begin{array}{c}\operatorname{Max}(\operatorname{Dim}) \\
109583\end{array}$ \\
\hline 20 & -15.58837 & 106 \\
40 & -15.588830 & 217 \\
60 & -15.58836 & 398 \\
80 & -15.58836 & 656 \\
\hline \hline
\end{tabular}

ing a single-particle energy term to the Hamiltonian, one that favors an oblate solution. The Hamiltonian of the model is

$$
H=-\chi Q \cdot Q-g P^{\dagger} P-\epsilon \sum_{m}|m| c_{j m}^{\dagger} c_{j m} .
$$

Because of the last term, the Hamiltonian is in general not rotationally invariant, so that its eigenstates do not have good angular momentum.

The first results we present are for ten particles in a $j$ $=25 / 2$ orbit. The dimension of the Hamiltonian matrix that would have to be diagonalized (in the $m$ scheme) for this problem is 109583 , which can be readily handled using the Lanczos algorithm.

In Table I, we present the results for $\chi=1, g=0$, and $\epsilon$ $=0.1$. The first row gives the exact ground-state energy; subsequent rows give the ground-state energy obtained using the DMRG approach as a function of $m$. At the end of each row, we show the maximum dimension Hamiltonian matrix that must be diagonalized in the DMRG procedure.

As is evident from the table, the results converge very rapidly to the exact ground-state energy. By $m=60$, we obtained a result that is off by only 1 part in $10^{6}$. For this choice of $m$, the largest matrix we had to diagonalize was $398 \times 398$. As a reminder, the reason that the matrices are so small is that we only need focus on those states in which the total angular momentum projection is zero and in which the number of particles equals the number of holes.

To see what happens in the presence of both quadrupole and pairing correlations, we next present the results for $\chi$ $=1, g=0.05$, and $\epsilon=0.1$. Such a value for the pairing strength leads to a strong depletion of probability from the hole levels, but still leaves a well defined Fermi surface.

The DMRG results for the ground-state energy in this case are plotted in Fig. 1 as a function of $m$. Included in the figure is an exponential fit to these results. When extrapolated, this exponential predicts an asymptotic end result of $-16.00358 \pm 0.00027$. This is in excellent agreement with the exact ground-state energy of -16.00367 , obtained using the Lanczos algorithm.

Table II shows results for the excitation energies of the lowest three excited states. The agreement for the excited states is almost as good as for the ground state, even though the method, as described earlier, only targeted the ground state in the optimization procedure.

The bottom line is that for these calculations, in which we could compare with the results of Lanczos diagonalization,

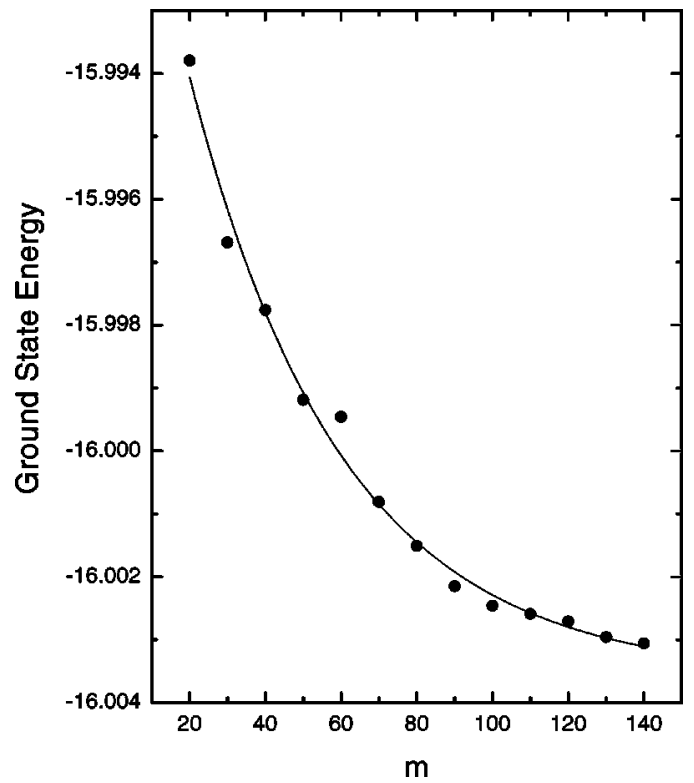

FIG. 1. Calculated results for the ground-state energy (circles) and an exponential fit to those results (solid curve) for a system of ten identical nucleons occupying a $j=25 / 2$ orbit and interacting via a Hamiltonian with parameters $\chi=1, g=0.05$, and $\epsilon=0.1$.

we obtain excellent agreement with the exact results, not just for the ground state but for higher excited states as well. Furthermore, the excellent results are achieved while diagonalizing matrices of moderate dimensions.

The fact that we could achieve a high level of accuracy not just for the ground state but for low-lying excited states as well may be a reflection that all these states have the same intrinsic structure. When different intrinsic structures enter, it may be necessary to modify our optimization criterion to include mixed density matrices that contain information on more than one state of the system [9].

The excellent quality of the results we obtained for a $j$ $=25 / 2$ orbit encouraged us to treat a more complex system, one for which exact diagonalization is not possible. We considered the case of a $j=55 / 2$ orbit with 20 particles. The other parameters of the calculation were $\chi=1, g=0.1$, and $\epsilon=0.2$. In this case, the exact calculation would involve a matrix of dimension $5.31064 \times 10^{13}$. The results for the ground state energy are shown in Table III. By $m=60$, the calculations have clearly converged and we should have a reliable ground-state energy to about six significant figures.

TABLE II. Excitation energies for ten particles in a $j=25 / 2$ level. The Hamiltonian parameters are $x=1, g=0.05$, and $\epsilon=0.1$.

\begin{tabular}{|c|c|c|c|}
\hline $\begin{array}{c}m \\
\text { Exact }\end{array}$ & $\begin{array}{c}E_{1} \\
0.51643\end{array}$ & $\begin{array}{c}E_{2} \\
0.87141\end{array}$ & $\begin{array}{c}E_{3} \\
1.10294\end{array}$ \\
\hline 40 & 0.52464 & 0.96894 & 1.18556 \\
\hline 60 & 0.51774 & 0.90385 & 1.11528 \\
\hline 80 & 0.51854 & 0.88581 & 1.11353 \\
\hline 100 & 0.51817 & 0.88274 & 1.11432 \\
\hline 120 & 0.51772 & 0.88068 & 1.11115 \\
\hline 140 & 0.51743 & 0.87842 & 1.10942 \\
\hline
\end{tabular}


TABLE III. Ground-state energy for 20 particles in a $j=55 / 2$ level. The Hamiltonian parameters are $x=1, g=0.1$, and $\epsilon=0.2$.

\begin{tabular}{ccc}
\hline \hline$m$ & $E_{\text {g.s. }}$ & $\operatorname{Max}(\operatorname{Dim})$ \\
Exact & $?$ & $5.31 \times 10^{13}$ \\
\hline 20 & -103.98844 & 100 \\
30 & -103.99420 & 180 \\
40 & -103.99574 & 240 \\
50 & -103.99827 & 361 \\
60 & -103.99894 & 430 \\
\hline \hline
\end{tabular}

In our view, these calculations demonstrate very clearly the great promise of the DMRG method in nuclear structure. As long as there is a well-defined Fermi surface in the problem, the method leads to extremely accurate results not only for the ground state but for low-lying excited states as well. Furthermore, the fairly rapid convergence that typically arises as a function of $m$ suggests that the method can be used quite reliably for very large-scale calculations.

While the DMRG method described in this Rapid Communication works best when there is a well-defined Fermi surface, it can nevertheless be used when one does not exist. As an example, if we carry out a calculation for ten particles in a $j=25 / 2$ orbit with $\chi=1, g=0$, and $\epsilon=0$, we obtain with the method a ground-state energy of -5.153947 for $m=60,-5.157945$ for $m=80$, and -5.161021 for $m$ $=100$. The exact result from Lanczos diagonalization is -5.188175 .

It is worthwhile to expand briefly on the rationale for the assumption that all levels of the single-particle basis admit the same number of states. This assumption greatly facilitates implementation of an iterative scheme in which the addition of new levels can be readily accommodated with no change of formalism. Relaxation of this assumption may be feasible, but no doubt at a significant cost to computational simplicity.

Now that we have completed this first test of the methodology with such impressive success, we are planning to gradually expand the complexity of the problems we consider. Our ultimate goal of course is to use this method to treat very large-scale nuclear structure problems involving both neutrons and protons populating a set of nondegenerate single-particle levels and interacting via a general interaction.

This work was supported in part by the National Science Foundation under Grant No. PHY-9970749, by the Spanish DGI under Grant No. BFM2000-1320-C02-02, and by NATO under Grant No. PST.CLG.977000. One of the authors (S.P.) wishes to thank the Institute for Nuclear Theory at the University of Washington for its hospitality and the U.S. Department of Energy for partial support during the completion of this work.
[1] See, e.g., K. Heyde, The Nuclear Shell Model (SpringerVerlag, Berlin, 1990).

[2] E. Caurier et al., Phys. Rev. C 59, 2033 (1999).

[3] See, e.g., W. Haxton and G. J. Stephenson, Jr., Prog. Part. Nucl. Phys. 12, 409 (1984).

[4] See, e.g., X. Ji, B. H. Wildenthal, and M. Vallieres, Nucl. Phys. A492, 215 (1989); O. Castaños and J. P. Draayer, ibid. A491, 349 (1989).

[5] M. Honma, T. Mizusaki, and T. Otsuka, Phys. Rev. Lett. 77, 3315 (1996).
[6] M. Shimizu, T. Otsuka, T. Mizusaki, and M. Honma, Phys. Rev. Lett. 86, 1171 (2001).

[7] S. R. White, Phys. Rev. Lett. 69, 2863 (1992); Phys. Rev. B 48, 10345 (1993).

[8] J. Dukelsky and G. Sierra, Phys. Rev. Lett. 83, 172 (1999); Phys. Rev. B 61, 12302 (2000).

[9] S. R. White, Phys. Rep. 301, 187 (1998).

[10] R. W. Richardson and N. Sherman, Nucl. Phys. 52, 221 (1964). 